\title{
Correction to: Prognostic Factors in Overall Survival of Patients with Unresectable Intrahepatic Cholangiocarcinoma Treated by Means of Yttrium-90 Radioembolization: Results in Therapy-Naïve Patients
}

\author{
P. Reimer ${ }^{1}$ - M. K. Virarkar $^{2}(1)$ M. Binnenhei ${ }^{3}$ - C. Justinger ${ }^{4}$ - M. R. Schön ${ }^{4}$ \\ K. Tatsch ${ }^{5}$
}

Published online: 29 January 2018

(C) Springer Science+Business Media, LLC, part of Springer Nature and the Cardiovascular and Interventional Radiological Society of Europe (CIRSE) 2018

\section{Correction to: Cardiovasc Intervent Radiol https://doi.org/10.1007/s00270-017-1871-2}

The published article has an error in the first name initial of one of the authors. "M. Justinger" should be "C. Justinger" as shown in this erratum.

The original article can be found online at https://doi.org/10.1007/ s00270-017-1871-2.

P. Reimer

Peter.Reimer@partner.kit.edu

1 Institute for Diagnostic and Interventional Radiology, Klinikum Karlsruhe, Academic Teaching Hospital of the University of Freiburg, Moltkestraße 90, 76133 Karlsruhe, Germany

2 Department of Diagnostic Radiology, M D Anderson Cancer Center, Houston, TX, USA

3 Medical Clinic III, Department of Hematology, Oncology, Infectious Disease and Palliative Medicine, Klinikum Karlsruhe, Academic Teaching Hospital of the University of Freiburg, Karlsruhe, Germany

4 Department of General and Visceral Surgery, Klinikum Karlsruhe, Academic Teaching Hospital of the University of Freiburg, Karlsruhe, Germany

5 Department of Nuclear Medicine, Klinikum Karlsruhe, Academic Teaching Hospital of the University of Freiburg, Karlsruhe, Germany 